\title{
Noncommutative and Non-Anticommutative Quantum Field Theory
}

\author{
J. W. Moffat \\ Department of Physics, University of Toronto, Toronto, Ontario M5S 1A7, Canada
}

\begin{abstract}
A noncommutative and non-anticommutative quantum field theory is formulated in a superspace, in which the superspace coordinates satisfy noncommutative and non-anticommutative relations. A perturbative scalar field theory is investigated in which only the non-anticommutative algebraic structure is kept, and one loop diagrams are calculated and found to be finite due to the damping caused by a Gaussian factor in the propagator.
\end{abstract}

\section{Introduction}

Noncommutative geometry is characterized by $d$ noncommuting self-adjoint operators $\hat{x}^{\mu}$ in a Hilbert space $\mathcal{H}$ satisfying $[1$, , 2]

$$
\left[\hat{x}^{\mu}, \hat{x}^{\nu}\right]=i \theta^{\mu \nu}
$$

where $\theta^{\mu \nu}$ is a non-degenerate $d \times d$ antisymmetric matrix. The product of two operators $\hat{\phi}_{1}$ and $\hat{\phi}_{2}$ has a corresponding Moyal $\star$-product

$$
\left(\hat{\phi}_{1} \star \hat{\phi}_{2}\right)(x)=\left[\exp \left(\frac{i}{2} \theta^{\mu \nu} \frac{\partial}{\partial \xi^{\mu}} \frac{\partial}{\partial \zeta^{\nu}}\right) \phi_{1}(x+\xi) \phi_{2}(x+\zeta)\right]_{\xi=\zeta=0} .
$$

Recently, the consequences for gravitation theory of using the Moyal $\star$-product to define a general quantum gravity theory on a noncommutative spacetime were analyzed, and it was found that a complex symmetric metric (non-Hermitian) theory could possibly provide a consistent underlying geometry on a complex coordinate manifold [3]. In a subsequent paper [4] the gravitational Einstein-Hilbert action given on a noncommutative spacetime geometry was expanded about a flat Minkowski spacetime, and the products of the fields were replaced by Moyal $\star-$ products. It was found that the planar one loop graviton graph and vacuum polarization are essentially the same as for the commutative perturbative result, while 
the non-planar graviton loop graph is damped due to the oscillatory behavior of the noncommutative phase factor in the Feynman integrand. Thus, the overall noncommutative perturbative quantum gravity theory remains unrenormalizable and divergent. The free-field theory in noncommutative field theories is the same as the commutative field theories, so only the interactions contain new information. Thus, all the planar graphs at all orders of perturbation theory are the same as in the commutative theory, except for unimportant vertex phase factors that depend only on the external momenta. There is no non-trivial dependence on internal momenta phase factors, which could help to dampen the divergent behavior of planar diagram loop integrals.

In the following, we shall investigate a spacetime geometry in which the spacetime coordinates are treated as 'superspace' vector operators in a superspace with 'fermionic' degrees of freedom, as well as the familiar 'bosonic', commuting or noncommuting degrees of freedom. A calculation of the one loop diagrams is carried out in scalar field theory. The dynamics of Yang-Mills gauge field theories and perturbative quantum gravity will be studied in a future publication.

\section{Superspace Formalism}

We shall formulate our quantization of spacetime on a superspace [5, 6] based on an extended Grassmann algebra $\mathcal{A}$. Let us define the superspace coordinates

$$
\rho^{\mu}=x^{\mu}+\beta^{\mu}
$$

where $x^{\mu}$ denote the familiar spacetime coordinates saisfying

$$
\left[x^{\mu}, x^{\nu}\right]=0
$$

and $\beta^{\mu}$ are coordinates in an associative Grassman algebra which satisfy

$$
\left\{\beta^{\mu}, \beta^{\nu}\right\} \equiv \beta^{\mu} \beta^{\nu}+\beta^{\nu} \beta^{\mu}=0
$$

Here, $\mu=0 \ldots 3$ although the formalism can easily be extended to higher-dimensional spaces.

The product of two operators $\hat{\phi}_{1}$ and $\hat{\phi}_{2}$ in our superspace is given by the oproduct

$$
\left(\hat{\phi}_{1} \circ \hat{\phi}_{2}\right)(\rho)=\left[\exp \left(\frac{1}{2} \omega^{\mu \nu} \frac{\partial}{\partial \rho^{\mu}} \frac{\partial}{\partial \eta^{\nu}}\right) \phi_{1}(\rho) \phi_{2}(\eta)\right]_{\rho=\eta}
$$




$$
=\phi_{1}(\rho) \phi_{2}(\rho)+\frac{1}{2} \omega^{\mu \nu} \frac{\partial}{\partial \rho^{\mu}} \phi_{1}(\rho) \frac{\partial}{\partial \rho^{\nu}} \phi_{2}(\rho)+O\left(\omega^{2}\right)
$$

where $\omega^{\mu \nu}$ is a nonsymmetric tensor

$$
\omega^{\mu \nu}=-\tau^{\mu \nu}+i \theta^{\mu \nu}
$$

with $\tau^{\mu \nu}=\tau^{\nu \mu}$ and $\theta^{\mu \nu}=-\theta^{\nu \mu}$. Moreover, $\omega^{\mu \nu}$ is Hermitian symmetric $\omega^{\mu \nu}=\omega^{\dagger \mu \nu}$, where $\nmid$ denotes Hermitian conjugation.

Let us define the notation

$$
\left[\phi_{1}(\rho), \phi_{2}(\rho)\right]_{\circ} \equiv \phi_{1}(\rho) \circ \phi_{2}(\rho)-\phi_{2}(\rho) \circ \phi_{1}(\rho)
$$

and

$$
\left\{\phi_{1}(\rho), \phi_{2}(\rho)\right\}_{\circ} \equiv \phi_{1}(\rho) \circ \phi_{2}(\rho)+\phi_{2}(\rho) \circ \phi_{1}(\rho) .
$$

From (3) and (6), we obtain for the superspace operator $\hat{\rho}$ :

$$
\begin{gathered}
{\left[\hat{\rho}^{\mu}, \hat{\rho}^{\nu}\right]_{\circ}=2 \beta^{\mu} \beta^{\nu}+i \theta^{\mu \nu}+O\left(\theta^{2}\right),} \\
\left\{\hat{\rho}^{\mu}, \hat{\rho}^{\nu}\right\}_{\circ}=2 x^{\mu} x^{\nu}+2\left(x^{\mu} \beta^{\nu}+x^{\nu} \beta^{\mu}\right)-\tau^{\mu \nu}+O\left(\tau^{2}\right) .
\end{gathered}
$$

If we now choose the limit in which $\beta^{\mu} \rightarrow 0$ and $\tau^{\mu \nu} \rightarrow 0$, then we get from (10) and (111):

$$
\begin{gathered}
{\left[\hat{\rho}^{\mu}, \hat{\rho}^{\nu}\right]_{\circ} \rightarrow\left[\hat{x}^{\mu}, \hat{x}^{\nu}\right]=i \theta^{\mu \nu},} \\
\left\{\hat{\rho}^{\mu}, \hat{\rho}^{\nu}\right\}_{\circ} \rightarrow\left\{\hat{x}^{\mu}, \hat{x}^{\nu}\right\}=2 x^{\mu} x^{\nu} .
\end{gathered}
$$

We see that (12) and (13) give us back the usual noncommutative expressions for the coordinate operators $\hat{x}^{\mu}$.

In the limit $x^{\mu} \rightarrow 0$ and $\theta^{\mu \nu} \rightarrow 0$, we get from (10) and (11):

$$
\begin{gathered}
{\left[\hat{\rho}^{\mu}, \hat{\rho}^{\nu}\right]_{\circ} \rightarrow\left[\hat{\beta}^{\mu}, \hat{\beta}^{\nu}\right]=2 \beta^{\mu} \beta^{\nu},} \\
\left\{\hat{\rho}^{\mu}, \hat{\rho}^{\nu}\right\}_{\circ} \rightarrow\left\{\hat{\beta}^{\mu}, \hat{\beta}^{\nu}\right\}=-\tau^{\mu \nu} .
\end{gathered}
$$

Let us now perform a similarity transformation on $\hat{\beta}^{\mu}$ to the Dirac $\gamma^{\mu}$ matrices, and choose the canonical diagonalized form $\tau^{\mu \nu}=2 \eta^{\mu \nu}$. We now obtain from (15):

$$
\left\{\hat{\beta}^{\mu}, \hat{\beta}^{\nu}\right\}=\left\{\gamma^{\mu}, \gamma^{\nu}\right\}=-2 \eta^{\mu \nu}
$$

where the $\gamma^{\mu}$ form a Clifford algebra $\mathcal{A}_{\gamma}$ over the real numbers $\mathcal{R}$ [7, 8$]$. 
In the following, we shall consider the simpler geometry determined by $\theta^{\mu \nu}=0$. We define a $\diamond$-product

$$
\begin{aligned}
& \left(\hat{\phi}_{1} \diamond \hat{\phi}_{2}\right)(\rho)=\left[\exp \left(-\frac{1}{2} \tau^{\mu \nu} \frac{\partial}{\partial \rho^{\mu}} \frac{\partial}{\partial \eta^{\nu}}\right) \phi_{1}(\rho) \phi_{2}(\eta)\right]_{\rho=\eta} \\
& =\phi_{1}(\rho) \phi_{2}(\rho)-\frac{1}{2} \tau^{\mu \nu} \frac{\partial}{\partial \rho^{\mu}} \phi_{1}(\rho) \frac{\partial}{\partial \rho^{\nu}} \phi_{2}(\rho)+O\left(\tau^{2}\right) .
\end{aligned}
$$

We now have

$$
\left\{\hat{\rho}^{\mu}, \hat{\rho}^{\nu}\right\}=2 x^{\mu} x^{\nu}+2\left(x^{\mu} \beta^{\nu}+x^{\nu} \beta^{\mu}\right)-\tau^{\mu \nu}
$$

\section{$3 \quad$ Non-Anticommutative Field Theory}

Our superspace formulation is a unified description of both noncommutative and non-anticommutative operator algebras in a Hilbert space $\mathcal{H}$. We could formulate a general field theory using the o-product of fields, which would contain complex exponential phase factors from the contributions associated with the antisymmetric tensor $\theta^{\mu \nu}$, as well as the real exponential factors associated with the contributions from the symmetric tensor $\tau^{\mu \nu}$. However, in the following, we shall restrict ourselves to the simpler case when $\theta^{\mu \nu}=0$.

For the $\diamond$-product of exponential functions, we have the rule

$$
\exp (i k \rho) \diamond \exp (i q \rho)=\exp [i(k+q) \rho] \exp \left[\frac{1}{2}(k \tau q)\right]
$$

where $(k \tau q) \equiv k_{\mu} \tau^{\mu \nu} q_{\nu}$. We have for two operators $\hat{f}$ and $\hat{g}$ :

$$
(\hat{f} \diamond \hat{g})(\rho)=\frac{1}{(2 \pi)^{8}} \int d^{4} k d^{4} q \tilde{f}(k) \tilde{g}(q) \exp \left[\frac{1}{2}(k \tau q)\right] \exp [i(k+q) \rho]
$$

where

$$
\tilde{f}(k)=\frac{1}{(2 \pi)^{4}} \int d^{4} \rho \exp (-i k \rho) f(\rho) .
$$

The product rule satisfies the associative rule

$$
(f \diamond g) \diamond h(\rho)=f \diamond(g \diamond h)(\rho) .
$$

We shall assume that derivatives act trivially in our space: $\partial / \partial \rho^{\mu}=\partial_{\mu}$ and

$$
\left[\rho^{\mu}, \partial_{\nu}\right]=-\eta_{\mu \nu}, \quad\left[\partial_{\mu}, \partial_{\nu}\right]=0
$$


In contrast to the noncommutative field theories, the kinetic energy component of the action in non-anticommutative field theories is not trivially the same as the commutative field theories, because of the symmetry of the tensor $\tau^{\mu \nu}$. The action for the scalar field $\phi$ is

$$
S=\int d^{4} \rho\left[\frac{1}{2} \partial_{\mu} \phi(\rho) \diamond \partial^{\mu} \phi(\rho)-\frac{m^{2}}{2} \phi(\rho) \diamond \phi(\rho)-V_{\diamond}(\phi)\right]
$$

where $V_{\diamond}(\phi)$ is the scalar field potential. For our calculations we shall choose the potential:

$$
V_{\diamond}(\phi)=\frac{\lambda}{4 !} \phi \diamond \phi \diamond \phi \diamond \phi
$$

In the noncommutative and the non-anticommutative cases there is an ambiguity in applying the quantization procedure in position superspace. The usual quantization condition

$$
[\phi(\vec{\rho}, t), \pi(\vec{\eta}, t)]=i \delta^{(3)}(\vec{\rho}-\vec{\eta})
$$

is defined for $\phi$ and $\pi$ in different superspace points, while the $\star$ and $\diamond$-products only make sense when the products are computed at the same superspace point. We can avoid this problem by working only in momentum space when performing Feynman rule calculations to obtain matrix elements. With this in mind, we shall formally define a $\triangle$-product of field operators $\hat{f}(\rho)$ and $\hat{g}(\eta)$ at different superspace points $\rho$ and $\eta$ :

$$
\hat{f}(\rho) \triangle \hat{g}(\eta)=\exp \left(-\frac{1}{2} \tau^{\mu \nu} \frac{\partial}{\partial \rho^{\mu}} \frac{\partial}{\partial \eta^{\nu}}\right) f(\rho) g(\eta)
$$

This product reduces to the $\diamond$-product of $\hat{f}$ and $\hat{g}$ in the limit $\rho \rightarrow \eta$.

For the $\diamond$-product rule in momentum space, we have the quantization condition

$$
[\tilde{\phi}(k), \tilde{\pi}(q)]_{\diamond}=i \delta^{(3)}(k-q) \exp \left[\frac{1}{2}(k \tau q)\right]
$$

In the case of the non-anticommutative field theory the exponential factor is relevant, due to the symmetry of the tensor $\tau^{\mu \nu}$, whereas in the noncommutative field theory the corresponding phase factor $\exp \left[-\frac{i}{2}(k \theta q)\right]$ has no relevance because of the antisymmetry of the tensor $\theta^{\mu \nu}$ and the definition $(k \theta q) \equiv k_{\mu} \theta^{\mu \nu} q_{\nu}$.

Let us now consider the Feynman rules for the non-anticommutative scalar field theory. We shall begin by calculating the two-point propagator. We quantize the 
scalar field in the same manner as in commutative field theory. We choose the free field operator $\phi(\rho)$ to have the form

$$
\phi(\rho)=\frac{1}{\sqrt{2(2 \pi)^{3}}} \int \frac{d^{3} k}{k_{0}}\left[a(k) \exp (-i k \rho)+a^{\dagger}(k) \exp (i k \rho)\right],
$$

where the annihilation and creation operators satisfy

$$
\left[a(k), a\left(k^{\prime}\right)\right]=\left[a^{\dagger}(k), a^{\dagger}\left(k^{\prime}\right)\right]=0, \quad\left[a(k), a^{\dagger}\left(k^{\prime}\right)\right]=\delta^{(3)}\left(\vec{k}-\vec{k}^{\prime}\right) .
$$

We now find using the $\triangle$-product

$$
\begin{aligned}
& {[\phi(\rho), \phi(\eta)]_{\triangle} \equiv i \bar{\Delta}(\rho-\eta)=\exp \left(-\frac{1}{2} \tau^{\mu \nu} \frac{\partial}{\partial \rho^{\mu}} \frac{\partial}{\partial \eta^{\nu}}\right)[\phi(\rho), \phi(\eta)]} \\
& =\frac{1}{(2 \pi)^{3}} \int \frac{d^{3} k}{k_{0}} \exp [-i k(\rho-\eta)] \epsilon\left(k_{0}\right) \exp \left[\frac{1}{2}(k \tau k)\right] \delta\left(k^{2}-m^{2}\right)
\end{aligned}
$$

where $\epsilon\left(k_{0}\right)=k_{0} /\left|k_{0}\right|$ is $=+1$ if $k_{0}>0$ and -1 if $k_{0}<0$. Also, we have

$$
[\phi(\rho), \phi(\eta)]_{\triangle}=\phi(\rho) \triangle \phi(\eta)-\phi(\eta) \triangle \phi(\rho) .
$$

Moreover, we have

$$
[i \bar{\Delta}(\rho)]_{\mid \tau^{\mu \nu} \rightarrow 0}=i \Delta(\rho)
$$

where $\Delta$ is the familiar commutative theory Green's function

$$
i \Delta(\rho)=\frac{1}{(2 \pi)^{3}} \int \frac{d^{3} k}{k_{0}} \exp (-i k \rho) \epsilon\left(k_{0}\right) \delta\left(k^{2}-m^{2}\right) .
$$

The modified Feynman propagator $\bar{\Delta}_{F}$ is defined by the vacuum expectation value of the time-ordered $\triangle$-product

$$
\begin{gathered}
i \bar{\Delta}_{F}(\rho-\eta) \equiv\langle 0|T(\phi(\rho) \triangle \phi(\eta))| 0\rangle \\
=\frac{i}{(2 \pi)^{4}} \int \frac{d^{4} k \exp [-i k(\rho-\eta)] \exp \left[\frac{1}{2}(k \tau k)\right]}{k^{2}-m^{2}+i \epsilon} .
\end{gathered}
$$

In momentum space this gives

$$
i \bar{\Delta}_{F}(k)=\frac{i \exp \left[\frac{1}{2}(k \tau k)\right]}{k^{2}-m^{2}+i \epsilon}
$$


which reduces to the standard commutative field theory form for the Feynman propagator

$$
i \Delta_{F}(k)=\frac{i}{k^{2}-m^{2}+i \epsilon}
$$

in the limit $\left|\tau^{\mu \nu}\right| \rightarrow 0$.

The interaction part of the action gives

$$
\begin{aligned}
S_{\mathrm{int}}= & \frac{\lambda}{4 !} \int d^{4} \rho \phi \diamond \phi \diamond \phi \diamond \phi(\rho)=\frac{\lambda}{4 !} \int d^{4} k_{1} \ldots d^{4} k_{4} \exp \left[\frac{1}{2}\left(k_{1} \tau k_{2}\right)\right] \exp \left[\frac{1}{2}\left(k_{2} \tau k_{3}\right)\right] \\
& \exp \left[\frac{1}{2}\left(k_{3} \tau k_{4}\right)\right] \phi\left(k_{1}\right) \phi\left(k_{2}\right) \phi\left(k_{3}\right) \phi\left(k_{4}\right)(2 \pi)^{4} \delta^{(4)}\left(k_{1}+k_{2}+k_{3}+k_{4}\right) .
\end{aligned}
$$

From this result, we can deduce that the vertex factor for the scalar non-anticommutative theory is given by

$$
V\left(k_{1}, k_{2}, k_{3}, k_{4}\right)=\exp \left\{\frac{1}{2}\left[\left(k_{1} \tau k_{2}\right)+\left(k_{2} \tau k_{3}\right)+\left(k_{3} \tau k_{4}\right)\right]\right\} .
$$

The vertex function factor for a general diagram has the form

$$
V\left(k_{1}, \ldots, k_{n}\right)=\sum_{i, j} \exp \left[\frac{1}{2} C_{i j}\left(k_{i} \cdot k_{j}\right)\right],
$$

where $k_{i} \cdot k_{j}=k_{i \mu} \tau^{\mu \nu} k_{j \nu}$ and $C_{i j}$ is a matrix.

Our Feynman rules are: for every internal line we insert a modified Feynman propagator $\bar{\Delta}_{F}(k)$ and integrate over $k$ with the appropriate numerical factor. We associate with every diagram a vertex factor $V\left(p_{1}, \ldots, p_{n} ; k_{1}, \ldots, k_{n}\right)$ where the $p s$ and $k s$ denote the external and internal momenta of the diagram, respectively.

\section{One Loop Self-energy Contribution}

The basic part of the one loop diagram for the non-anticommutative scalar field theory with the potential (25) is given by

$$
\Sigma=\frac{\lambda}{2(2 \pi)^{4}} \int d^{4} k \frac{i \exp \left[\frac{1}{2}(k \tau k)\right]}{k^{2}-m^{2}+i \epsilon} .
$$

We now perform an analytic continuation in the complex $k$ and $p$ planes with $k^{2}=-q^{2}=-\left(q_{4}^{2}+q_{1}^{2}+q_{2}^{2}+q_{3}^{2}\right)=-\kappa^{2}$ where $q$ denotes the Euclidean momentum vector. Moreover, we have $p^{2}=-\rho^{2}=-\left(p_{4}^{\prime 2}+p_{1}^{\prime 2}+p_{2}^{\prime 2}+p_{3}^{\prime 2}\right)$. We treat $\tau^{\mu \nu}$ as 
a non-degenerate 'metric' with $\operatorname{det}\left(\tau^{\mu \nu}\right) \neq 0$, so that we can find an orthonormal basis $v_{1}, \ldots, v_{n}$ of the tangent space at each point such that $\tau\left(v_{\mu}, v_{\nu}\right)=0$ if $\mu \neq \nu$ and $\tau\left(v_{\mu}, v_{\nu}\right)= \pm C$, where $C$ is a constant. We choose the Lorentzian signature $\tau^{\mu \nu}=C \eta^{\mu \nu}$ and set $\frac{1}{2}(k \tau k) \equiv \frac{1}{2} k_{\mu} \tau^{\mu \nu} k_{\nu}=-\frac{\kappa^{2}}{\Lambda^{2}}$ and $\frac{1}{2}(k \tau p) \equiv \frac{1}{2} k_{\mu} \tau^{\mu \nu} p_{\nu}=-\frac{\kappa \rho}{\Lambda^{2}}$, where $\Lambda$ is a constant with the dimensions of energy. Since the numerator of $\bar{\Delta}_{F}(k)$ is an entire function, there are no additional singularities in the finite momentum plane that can cause problems for the analytic continuation and unitarity.

We get in Euclidean momentum space

$$
\begin{gathered}
\Sigma=\frac{\lambda}{16 \pi^{2}} \int_{0}^{\infty} d \kappa \frac{\kappa^{3} \exp \left(-\frac{\kappa^{2}}{\Lambda^{2}}\right)}{\kappa^{2}+m^{2}} \\
=\frac{\lambda}{32 \pi^{2}}\left[\Lambda^{2}-m^{2} \exp \left(\frac{m^{2}}{\Lambda^{2}}\right) E_{i}\left(\frac{m^{2}}{\Lambda^{2}}\right)\right],
\end{gathered}
$$

where $E_{i}(x)$ is the exponential integral

$$
E_{i}(x)=\int_{1}^{\infty} d t \frac{\exp (-x t)}{t}
$$

The self-energy contribution $\Sigma$ can be expanded for $\Lambda \gg m$ as

$$
\Sigma=\frac{\lambda}{32 \pi^{2}}\left\{\Lambda^{2}-m^{2}\left[\gamma+\ln \left(\frac{m^{2}}{\Lambda^{2}}\right)\right]+O\left(\frac{m^{2}}{\Lambda^{2}}\right)\right\}+O\left(\lambda^{2}\right) .
$$

When we include the part with the vertex factor, we get

$$
\tilde{\Sigma}(\rho)=\frac{\lambda}{16 \pi^{2}} \int_{0}^{\infty} d \kappa \frac{\kappa^{3} \exp \left[-\left(\frac{\kappa^{2}}{\Lambda^{2}}+\frac{3 \kappa \rho}{2 \Lambda^{2}}\right)\right]}{\kappa^{2}+m^{2}} .
$$

This integral is also convergent and yields a finite self-energy contribution.

The vertex corrections in the non-anticommutative $\lambda \phi_{\diamond}^{4}$ theory will take the form

$$
\Gamma(s)=\frac{\lambda}{2(2 \pi)^{4}} \int \frac{d^{4} k \exp \left\{\frac{1}{2}[(k \tau k)+(l \tau l)]\right\} V\left(p_{1}, p_{2}, p_{3}, p_{4}\right)}{\left(l^{2}-m^{2}+i \epsilon\right)\left(k^{2}-m^{2}+i \epsilon\right)},
$$

where $l=k-p$ and

$$
s \equiv p^{2}=\left(p_{1}+p_{2}\right)^{2}, \quad t=\left(p_{1}-p_{2}\right)^{2}, \quad u=\left(p_{1}-p_{4}\right)^{2}
$$

are the Mandelstam variables. Then, we have for the vertex correction contributions $\Gamma_{1}=\Gamma(s), \Gamma_{2}=\Gamma(t), \Gamma_{3}=\Gamma(u)$. When we transform this result into Euclidean 
momentum space, we find that the Gaussian exponential factor in the internal momentum $k$ yields convergent vertex corrections.

We see that in the non-anticommutative scalar field theory, the one loop selfenergy diagram is finite for a generic value of the external momentum $p$ and for fixed finite values of the parameter $\Lambda$. The same holds true for the vertex one loop corrections. When $\Lambda \rightarrow \infty$, we obtain the standard classical spacetime continuum theory and the one loop self-energy is quadratically divergent.

The convergence of both planar and non-planar loop diagrams should hold to all orders of perturbation theory. Thus, the self-energy corrections to the mass and the coupling constant denoted by $\delta m^{2}$ and $\delta \lambda$ will be finite and the 'renormalization' of the mass and the coupling constant will be a finite procedure.

An analysis of the unitarity condition for the non-anticommutative amplitudes shows that it can be satisfied, because the propagator $\bar{\Delta}_{F}$ is modified only by an entire function compared to the standard, commutative propagator [9]. Thus, the position of singularities in the finite region of the complex momentum plane are unaltered and the Cutkosky cutting rules can be satisfied. The amplitudes avoid essential singularities at infinity and the crossing symmetry relations are only physical at infinite energies, if we choose $\tau^{m n} \neq 0$ and $\tau^{00}=\tau^{0 n}=0(m, n=1,2,3)$. We can then choose an orthonormal frame such that $\tau^{m n}=-\delta^{m n} / \Lambda^{2}$, and the asymptotic behavior of the modified propagator as $p^{2} \rightarrow \pm \infty$ is

$$
\bar{\Delta}_{F} \sim \exp \left(-\frac{1}{2} \mathbf{p}^{2} / \Lambda^{2}\right)
$$

where $\mathbf{p}$ denotes the 3 -momentum vector and $\mathbf{p}^{2}>0$. This choice of orthonormal frame avoids the possibility of essential singularities in amplitudes at infinite energies. However, it breaks Lorentz invariance but the Lorentz symmetry can be broken 'softly' by adding a Higgs spontaneous symmetry breaking contribution to the action [10].

\section{Conclusions}

Classical spacetime geometry has been generalized to a superspace with coordinates that are, in general, both noncommutative and non-anticommutative, corresponding to their possessing 'bosonic' and 'fermionic' degrees of freedom. The Moyal $\star$-product has been extended to a o-product of field functions, defined in terms of a nonsymmetric Hermitian tensor $\omega^{\mu \nu}$, instead of the antisymmetric tensor $\theta^{\mu \nu}$ used in the Moyal $\star$-product. We studied the simpler geometry in which the antisymmetric tensor $\theta^{\mu \nu}=0$, and only the symmetric tensor $\tau^{\mu \nu}$ contributes to a $\diamond$-product 
of field operators. The free-field theory is different from the corresponding one in commutative field theory, resulting in a modified Feynman propagator $\bar{\Delta}_{F}(k)$ and a modified vertex function $V\left(k_{1}, \ldots, k_{n}\right)$.

We restricted ourselves to the case $\tau^{m n} \neq 0$ and $\tau^{00}=\tau^{0 n}=0$, because only then do we get a field theory that avoids potential problems with unitarity and causality. The same circumstances exist for the noncommutative field theories with the restriction that $\theta^{m n} \neq 0$ and $\theta^{0 n}=0$.

By calculating the one loop self-energy graph in $\lambda \phi_{\diamond}^{4}$ theory, we found that it is finite for both planar and non-planar graphs. This result should hold for all loop graphs due to the convergence of the loop diagrams, caused by the exponential Gaussian factor associated with the modified Feynman propagator.

The form of our modified Feynman propagator $\bar{\Delta}_{F}(k)$ involves an entire function of $k^{2}$. This means that the theory is nonlocal, although the severity of the nonlocality has been reduced due to the choice $\tau^{m n} \neq 0$ and $\tau^{00}=\tau^{0 n}=0$. A modified Feynman propagator of the form

$$
i \bar{\Delta}_{F}(k)=\frac{i \exp \left(\frac{k^{2}-m^{2}}{\Lambda^{2}}\right)}{k^{2}-m^{2}+i \epsilon}
$$

was used in the development of a nonlocal field theory, which was perturbatively finite and for Yang-Mills theory was gauge invariant to all orders [11, 12, 13]. However, this field theory lacked a motivation for such a modified propagator, whereas in the present scheme our quantization of spacetime, based on non-anticommuting coordinates in a superspace, provides a fundamental origin for the modified propagator and the convergence of loop integrals. Nonlocal field theories of this kind were studied extensively in the 1960s and 1970s [14] and have also been the subject of investigations in superstring theory and D-brane theory [15.

In view of the finiteness of our loop diagrams, we do not find any peculiar ultraviolet-infrared combination of divergences, as is found in the noncommutative field theories [16].

The introduction of noncommutative and non-anticommutative coordinates implies a natural unit of length $\ell$ in our superspace formalism. The introduction of this unit of length makes it possible to remove divergences in ordinary point particle, local field theory. To accomplish this, we have given up the idea of a continuous spacetime continuum, treating the coordinates of spacetime points as operators in a supervector space. The introduction of a smallest unit of length in spacetime, forces us to rescind the usual assumption of commutativity (or anticommutativity) of coordinates $x^{\mu}$, otherwise the assumption of Lorentz invariance of the spectra of the operators $\hat{x}^{\mu}$, if they commute, implies continuous spectra corresponding to the 
measurements of spacetime points.

It will be interesting to extend our investigation to gauge field theories such as Yang-Mills theory and quantum gravity. By treating quantum gravity as an effective perturbative theory [4, it is anticipated that due to the finite results for the loop graphs obtained in scalar field theory, there exists a strong possibility that a self-consistent, effective quantum gravity theory can be derived, based on a nonanticommutative spacetime geometry. This will be the subject of a future paper.

\section{Acknowledgments}

This work was supported by the Natural Sciences and Engineering Research Council of Canada. I thank Craig Burrell and Alex Williamson for helpful discussions.

\section{References}

[1] A. Connes, M. R. Douglas and A. Schwarz, JHEP 9802 (1998) 003, hepth/9711162; J. Madore, "An Introduction to Noncommutative Geometry and its Physical Applications", Cambridge University Press, 1995.

[2] N. Seiberg and E. Witten, JHEP 9909 (1999) 032, hep-th/9908142.

[3] J. W. Moffat, Phys. Lett. b491,345 (2000), hep-th/0007181.

[4] J. W. Moffat, Phys. Lett. B493, 142(2000), hep-th/0008089.

[5] B. S. De Witt, "Supermanifolds", Cambridge University Press, Cambridge, U. K., second edition, 1992.

[6] I. L. Buchbinder and S. M. Kuzenko, "Ideas and Methods of Supersymmetry and Supergravity", revised edition, Institute of Physics Publishing, Dirac House, Bristol, U. K. 1998.

[7] I. V. Tyutin, hep-th/0101068; hep-th/0101046 v2.

[8] F. Gaetano, math.QA/0002194 v2.

[9] J. W. Moffat, hep-th/0011229. 
[10] J. W. Moffat, Int. J. Mod. Phys. D2, 351 (1993), gr-qc/9211020; J. W. Moffat, Found. of Phys. 23, 411 (1993). gr-qc/9209001; J. W. Moffat, to be published in a volume dedicated to Hagen Kleinert on the occasion of his 60th birthday by World Scientific, Singapore. astro-ph/9811390.

[11] J. W. Moffat, Phys. Rev. D41, 1177 (1990).

[12] D. Evans, J. W. Moffat, G. Kleppe, and R. P. Woodard, Phys. Rev. D43, (1991).

[13] G. Kleppe and R. P. Woodard, Ann. of Phys. 221, 106 (1993).

[14] V. Ya. Fainberg and M. A. Soloviev, Ann. of Phys. 113, 421 (1978); M. Z. Iofa and V. Ya. Fainberg, Zh. Eksp. Teor. Fiz. 56, 1644 (1969) [Sov. Phys. JETP 29, 880 (1969)]; M. A. Soloviev, Theor. Math. Phys. 7, 183 (1971); ibid. 20, 299 (1974); F. Constantinescu and J. G. Taylor, J. Math. Phys. 15, 824 (1974); G. V. Efimov, Comm. Math. Phys. 5, 42 (1967).

[15] A. Kapustin, hep-th/9912044.

[16] A. Matusis, L. Susskind and N. Toumbas, hep-th/0002075; S. Minwalla, M. Van Raamsdonk and N. Seiberg, hep-th/9912072. 